\title{
Prevalencia de hipoacusia neurosensorial inducida por ruido en empresas del sector madera de la ciudad de Cartagena. 2015
}

\author{
Prevalence of sensorineural hearing loss induced noise in wood sector \\ companies city Cartagena.
}

Darío David Sierra Calderón¹, Elías Alberto Bedoya Marrugo²

\section{Resumen}

Objetivo: Determinar prevalencia de hipoacusia neurosensorial inducida por ruido en los trabajadores expuestos a ruido en cuatro empresas dedicadas al procesamiento de madera en la ciudad de Cartagena. Método: Se realizó un estudio descriptivo de corte transversal en cuatro empresas de aserríos del sector madera de la ciudad de Cartagena, dedicadas a la transformación de la troza de madera en tablas y listones a través de operaciones de corte, cepillado y canteado de la madera. La población objeto de estudio fue de veinte trabajadores del área de maquinado expuestos a ruido. Se hizo una evaluación preliminar para conocer en forma detallada el ambiente de trabajo y las circunstancias bajo las cuales se exponen los trabajadores al ruido, las características del ruido y sus fuentes. Resultados: La prevalencia de hipoacusia neurosensorial inducida por ruido fue del $20 \%$. Se encontró en el grupo objeto de estudio un $5 \%$ con hipoacusia entre trabajadores de 41 a 45 ańos de edad y un $15 \%$ en trabajadores de más de 46 años de edad. En cuanto al tiempo de servicio en las distintas empresas, los trabajadores de 1 a 30 años presentaron el $20 \%$ de hipoacusia neurosensorial inducida por el ruido. En la medición de la exposición al ruido basada por puesto de trabajo en las distintas empresas seleccionadas se determinó el nivel continuo equivalente que oscila entre $95,7 \mathrm{~dB}(\mathrm{~A})$ a 101,9 $\mathrm{dB}(\mathrm{A})$ el cual sobrepasa los valores límites permisibles de acuerdo a los criterios de ACGIH de USA. El nivel de riesgo es alto en una de las empresas mientras se encontró un nivel de riesgo medio en las otras empresas seleccionadas.

Palabras Clave: pérdida auditiva, ruido, aserraderos

\section{Abstract}

Objective: To find out prevalence of noise-induced sensorineural hearing loss in workers exposed to noise in four companies implicated in wood processing in the city of Cartagena. Method: A descriptive cross-sectional study was conducted in four companies sawmills the timber sector of the city of Cartagena, dedicated to the transformation of the log wooden board and batten through cutting, brushing and chamfering wood. The study population was twenty machining area workers exposed to noise. A preliminary evaluation was done to know in detail the work environment and the

${ }^{1}$ Docente investigador, Facultad de ingeniería, Programa de Seguridad e higiene ocupacional, Fundación Universitaria Tecnologico Comfenalco.

${ }^{2}$ Coordinador de investigación, Grupo CIPTEC, Facultad de ingeniería, Programa de Seguridad e higiene ocupacional, Fundación Universitaria Tecnológico Comfenalco.

Correspondencia: eabedoya8@gmail.com

Darío David Sierra Calderón: http://orcid.org/0000-0002-0113-5594, Elías Alberto Bedoya Marrugo: http://orcid.org/0000-0002-2931-9600

Recibido: 03-03-2015 Aceptado:12-05-2016 
circumstances under which workers are exposed to noise, noise characteristics and sources. Results: The prevalence of noise-induced sensorineural hearing loss was 20\%. It was found in the study group that $5 \%$ with hearing loss among workers 41 to 45 years old and $15 \%$ in workers over 46 years old. As for the time of service in the various companies, workers from 1 to 30 years had $20 \%$ of sensorineural hearing loss induced by noise. In the measurement of noise exposure per job based on the different selected companies the equivalent continuous level of between $95.7 \mathrm{~dB}(\mathrm{~A})$ to $101.9 \mathrm{~dB}(\mathrm{~A})$ which exceeds the permissible limit values determined according to the US ACGIH criteria. The risk level is high in one of the companies as a medium risk was found in the other selected companies.

Keywords: hearing loss, noise, sawmills

\section{Introducción}

El ruido se entiende como aquella dispersión sonora desagradable al oído y por ende se califica como indeseable. Aunque es necesario entender que no todos los sonidos logran ser percibidos por el oído humano (1).

Como riesgo ocupacional, el ruido es reconocido desde la antigüedad. Ahora, eventos como el aumento de las máquinas y su uso en las fábricas (aportando mayor energía y velocidad), remplazarían la fuerza del hombre (el cual queda en desventaja); agregando el factor ruido y su exposición, como nuevo componente de riesgo ocupacional propenso de generar alteraciones de la salud en los trabajadores (2). Aunque existen actividades laborales con exposición por debajo de los niveles permisibles, generan algún tipo de afecciones, debido a la cronicidad y continuidad frente al riesgo (3).

El ruido es visto como un peligroso agente contaminante (4), gran generador de daños al sistema auditivo, que además altera otros sistemas, disminuyendo el aprovechamiento de la labor del trabajador y a la postre mermando la productividad de organización (5). Conforme a cifras entregadas por la Organización Mundial de la Salud en el año 2002, se detectaron casos alrededor del 16\% de casos confirmados de pérdida de audición laboral alrededor del mundo (6). Como enfermedad ocupacional prevalente de aspectos del trabajo se puede referenciar a la pérdida auditiva o hipoacusia neurosensorial inducida por ruido (HNIR) como afección predominantemente entre personal expuesto.

Otras investigaciones demuestran afecciones más relevantes entre su grupo de expuestos al reportar cifras superiores donde se ha afectado la salud de los trabajadores al tener 78,5\% de casos positivos de hipoacusia atribuible al ruido ocupacional (7). Investigaciones con determinantes para reconocer la exposición y afectación provocada por ruido del $62.5 \%$, detectaron niveles de ruidos igual o superior a $85 \mathrm{~dB}-\mathrm{A}$, pero con el agravante de no utilizar dispositivos de protección para ruidos en el $96,3 \%$ de los trabajadores y, el $62.2 \%$ con más de 10 años de exposición a ruidos y $20.8 \%$ sin respuesta a intensidades de $25 \mathrm{~dB}, 50.0 \%$ a intensidades de $40 \mathrm{~dB}$ y $729.2 \%$ no respondieron a estímulos sonoros de $60 \mathrm{~dB}$, con clara afectación auditiva permanente (8).

La Oficina de Estadísticas Laborales de los Estados Unidos identificó diversos eventos de salud reportados, entre los cuales la pérdida auditiva ocupacional es vista como una de las enfermedades de carácter laboral con mayor frecuencia y aparición en el sector manufacturero, donde laboran un aproximado de 16 millones de personas, entre los cuales se han reportado 17,700 casos de pérdida auditiva por cada 59,100 casos generales, manejando una razón de 1 
caso de pérdida auditiva por cada nueve enfermedades notificarles (9).

Referentes internacionales han indicado que en países como India, existen prevalencias por pérdida de la audición en el orden del $28 \%$, específicamente en relación con el manejo de la sierra circular y mesas tipo aserrado donde asciende hasta el $28 \%$ con exposición a niveles de ruido promedio $90.2 \mathrm{~dB}$, suficientes para generar daño en el sistema auditivo, que presenta exposiciones superiores a las 8 horas/día (10).

Estudios en occidente demuestran otra relación entre la exposición al riesgo sin utilización de equipos de protección: $53 \%$ de los trabajadores mantuvo audición normal, mientras que la disminución auditiva tuvo lugar en el restante $47 \%$ de los trabajadores. En estos casos las zonas de trabajo presentaron niveles de ruido en un rango de 83 a 102 decibeles (11). La Organización Mundial de la Salud durante el ańo 2002 ya había revelado que cerca del 16\% de los casos de pérdida de audición registrados en el mundo son adquiridos en el lugar de trabajo. De acuerdo con la Oficina de Estadísticas Laborales de los Estados Unidos, la pérdida auditiva ocupacional es la enfermedad laboral reportada con más frecuencia en el sector manufacturero. En Colombia, para el año 2008, se reportó la presencia de hipoacusia neurosensorial inducida por ruido en un 4,5\%, convirtiéndola para ese año en la enfermedad más diagnosticada, después de las enfermedades osteomusculares (12).

Se ha confirmado que trabajadores expuestos al ruido presentan audiometrías compatibles con lesiones auditivas por ruido, de los que más de la mitad presentaban afectación de las frecuencias conversacionales, factibles de ser evitadas mediante el uso de los equipos de protección individual debido a la escasa percepción del riesgo por parte de los trabajadores. Se demostró, además, como factores decisivos para la manifestación de la pérdida de la audición el tiempo de exposición y la edad (13). Particularizando los casos a tratar en este artículo, otro estudio llevado a cabo por la Universidad de Valencia en permitió determinar los niveles promedio de exposición diaria a presión sonora en distintos puestos de trabajo en la industria de la madera, estudio que permitió encontrar niveles de ruido que en el $28 \%$ de los casos, sobrepasaba los $85 \mathrm{~dB}$, límite actual y en un 7\%, superó los $90 \mathrm{~dB}$, que era el límite de exposición establecido según la legislación de 1995 para esa época (13). Al tratar sobre aspectos de la sintomatología general de los efectos del ruido en personas que laboran, las causas de pérdida de audición, la hipoacusia neurosensorial inducida por ruido es uno de los problemas más comunes a los que se enfrenta el otorrinolaringólogo y el médico general y con no muy buenas expectativas para el paciente (15).

Se debe considerar que conforme estudios sobre el ruido ocupacional, éste puede ser un factor determinante en el desarrollo de hipertensión arterial en trabajadores expuestos con una significativa incidencia de casos en el orden de (>50\%) en áreas donde el nivel de ruido se haya por encima del rango normal (16). La mayoría de los trabajadores empleados en la industria de la madera se han venido exponiendo desde mucho tiempo a niveles de ruido críticos para su salud y en algunos puestos de trabajo estos superan los $100 \mathrm{~dB}$, lo cual los hace susceptibles a los anteriores síntomas mencionados (17). Estudios donde el ruido hace aparición en la industria procesadora de madera, caracterizada por los procesos manuales de traslado, donde hay esfuerzo físico de moderado a intenso, presencia de altas temperaturas y elevados niveles de ruido, han demostrado incidencia en altos niveles de presión arterial y alteraciones de frecuencia cardíaca, sumado al detrimento de la salud por pérdida de la capacidad auditiva (18). Entre las enfermedades prevalentes a nivel laboral se encuentra la pérdida auditiva o hipoacusia neurosensorial inducida por ruido 
(HNIR). El ruido tipificado como contaminante en escenarios laborales tiene la capacidad de dañar al sistema auditivo y también puede afectar otros sistemas con impacto negativo en el rendimiento del trabajador y con el tiempo en la productividad de la empresa (19-20). Como principal objetivo de este estudio tiene el determinar la prevalencia de hipoacusia neurosensorial inducida o por ruido entre los trabajadores de las empresas estudiadas.

\section{Metodología}

Se realizó un estudio descriptivo de corte transversal en cuatro empresas de aserríos del sector madera de la ciudad de Cartagena, dedicadas a la transformación de la troza de madera en tablas y listones a través de operaciones de corte, cepillado y canteado de la madera.

La población fue integrada por 20 trabajadores del área de maquinado expuestos a ruido.

Se realizó una evaluación preliminar para conocer en forma detallada las condiciones de trabajo en las cuales los trabajadores se exponen al ruido en las distintas operaciones del proceso de aserrado de la madera.

En la medición de la exposición al ruido se utilizaron las técnicas y estrategias establecidas por el Instituto Nacional de Seguridad e Higiene de Espańa y la GATI-HNIR del Ministerio de Seguridad Social. Los instrumentos utilizados fueron dosímetro marca QUEST, modelo EG5, y el Sonómetro marca QUEST Tipo II, Sound Pro DL. Con las lecturas registradas se determinó el nivel continuo equivalente diario y el nivel de pico, para luego determinar el nivel de riesgo a la exposición al ruido teniendo en cuenta los valores límites permisibles de ACGHI de Estados Unidos. En el diagnóstico de la hipoacusia neurosensorial inducida por ruido, se aplicó un cuestionario, pre- vio consentimiento voluntario, a los trabajadores expuestos a ruido en su área de trabajo. El instrumento recogió datos demográficos tales como: edad, antigüedad en la empresa y antigüedad en el puesto; también recopiló información relacionada con la disminución de la capacidad auditiva: nivel de ruido por puestos de trabajo, uso de equipo de protección auditiva, exposición a golpes en la cabeza. Se realizaron entrevistas a los trabajadores para realizar historias clínicas ocupacionales, exámenes físicos con otoscopia y exámenes paraclínicos especialmente audiometrías vía aérea y ósea con la ayuda de un audiómetro marca Veltone, teniendo en cuenta los requisitos presentes en la GATI-HNIR del Ministerio de Seguridad Social con el fin de identificar pacientes con hipoacusia neurosensorial inducida por ruido. Se tuvieron en cuenta los requerimientos legales con respecto al manejo confidencial de la información y el tiempo de retención de los registros.

\section{Resultados}

\section{Caracterización de las condiciones de trabajo}

En las cuatro empresas seleccionadas realizan el proceso del aserrado de la madera, que consiste en dividir la troza de la madera en tablas y listones, según el uso que se le vayan a dar los clientes. Inicialmente la troza es cortada a través de la sierra sinfín y circular. En esta operación participan dos trabajadores por cada máquina en la cual se exponen a ruido variado durante su jornada. El puesto de trabajo permite la movilidad y realizan pocas tareas en la operación. Posteriormente se le da el acabo final por medio de la cepilladora y la canteadora. En esta operación participan cuatro trabajadores. Los puestos de trabajo son móviles con pocas tareas en la operación y el tipo de ruido es variado durante su jornada de ocho horas y 48 horas semanales. No se dispone de ningún control de ingeniería al ruido y los trabajadores no utilizan ningún tipo de protección auditiva personal. 
Evaluación de la exposición ocupacional al ruido

En la medición de la exposición al ruido basada por puesto de trabajo en las distintas empresas seleccionadas se determinó que el nivel continuo equivalente diario oscila entre $95,7 \mathrm{~dB}(\mathrm{~A})$ y 101,9 $\mathrm{dB}(\mathrm{A})$ que sobrepasa los valores límites permisibles de acuerdo a los criterios de ACGIH de USA. Su nivel de riesgo es alto en la empresa 1 y nivel de riesgo medio en las otras empresas seleccionadas.

Tabla 1. Nivel de riesgo a la exposición al ruido

\begin{tabular}{|c|c|c|c|c|c|c|c|c|c|c|c|c|c|c|}
\hline $\begin{array}{c}\text { Nombres de las } \\
\text { Empresas }\end{array}$ & Lugar de Medición & $\begin{array}{l}\text { Nombres } \\
\text { Trabajadores }\end{array}$ & $\begin{array}{l}\text { Tipo de } \\
\text { Ruido }\end{array}$ & $\begin{array}{c}\text { Tiempo } \\
\text { Exposición }\end{array}$ & $\underset{\text { ACGIH }}{\text { TLVs dB(A). }}$ & $\begin{array}{c}\text { Nivel Continuo } \\
\text { Equivalente (Leq) }\end{array}$ & \begin{tabular}{|c|} 
Nivel \\
Continuo \\
Diario \\
$\begin{array}{c}\text { Equivalente } \\
\text { (Leq,d) }\end{array}$ \\
\end{tabular} & $\begin{array}{c}\text { Nivel Presión } \\
\text { Sonora Minimo } \\
\text { (Lasmn) }\end{array}$ & \begin{tabular}{|c|} 
Nivel Presión \\
Sonora \\
Máximo \\
(Lasmx)
\end{tabular} & $\begin{array}{c}\text { Promedio } \\
\text { ponderado de } \\
\text { tiempo (TWA) }\end{array}$ & $\begin{array}{c}\text { ProjectedTW } \\
\text { A (8:00) }\end{array}$ & $\begin{array}{l}\text { Nivel Pico } \\
\text { (Lcpk) }\end{array}$ & NPA dB(A) & $\begin{array}{l}\text { NIVEL DEL } \\
\text { RIESGO }\end{array}$ \\
\hline \multirow[b]{2}{*}{ Empresa \#1 } & Cepillado/Canteado & $\begin{array}{l}\text { Trabajador \#1 } \\
\text { Trabajador \#2 }\end{array}$ & Intermitente & 8 horas & $85 \mathrm{~dB}(\mathrm{~A})$ & $101,7 \mathrm{~dB}(\mathrm{~A})$ & $101,9 \mathrm{~dB}(\mathrm{~A})$ & $61 \mathrm{~dB}(\mathrm{~A})$ & $121,7 \mathrm{~dB}(\mathrm{~A})$ & $101,9 \mathrm{~dB}(\mathrm{~A})$ & $101,7 \mathrm{~dB}(\mathrm{~A})$ & $136,6 \mathrm{~dB}$ & $>100 \mathrm{~dB}(\mathrm{~A})$ & ALTO \\
\hline & Sierra Sinfin & $\begin{array}{l}\text { Trabajador \#3 } \\
\text { Trabajador \#4 }\end{array}$ & Intermitente & 8 horas & $85 \mathrm{~dB}(\mathrm{~A})$ & $96 \mathrm{~dB}(\mathrm{~A})$ & $96,1 \mathrm{~dB}(\mathrm{~A})$ & $64,3 \mathrm{~dB}$ & $116,7 \mathrm{~dB}$ & $96,1 \mathrm{~dB}$ & $96 \mathrm{~dB}$ & $137 \mathrm{~dB}$ & $\begin{array}{l}85 \mathrm{~dB}(\mathrm{~A}) \mathrm{a} \\
99,5 \mathrm{~dB}(\mathrm{~A})\end{array}$ & MEDIO \\
\hline \multirow{4}{*}{ Empresa \#2 } & Sierra Sinfin \#1 & $\begin{array}{l}\text { Trabajador \#1 } \\
\text { Trabajador \#2 }\end{array}$ & Intermitente & 8 horas & $85 \mathrm{~dB}(\mathrm{~A})$ & $92,7 \mathrm{~dB}(\mathrm{~A})$ & $91,8 \mathrm{~dB}(\mathrm{~A})$ & $60 \mathrm{~dB}(\mathrm{~A})$ & $114 \mathrm{~dB}(\mathrm{~A})$ & $91,7 \mathrm{~dB}(\mathrm{~A})$ & $92,7 \mathrm{~dB}(\mathrm{~A})$ & $133,1 \mathrm{~dB}(\mathrm{~A})$ & $\begin{array}{l}85 \mathrm{~dB}(\mathrm{~A}) \mathrm{a} \\
99,5 \mathrm{~dB}(\mathrm{~A})\end{array}$ & $\begin{array}{l}\text { MEDIO } \\
----\end{array}$ \\
\hline & Sierra Sinfin \#2 & $\begin{array}{l}\text { Trabajajāor \#3 } \\
\text { Trabajador \#4 }\end{array}$ & Intermitente & 8 horas & $85 \mathrm{~dB}(\mathrm{~A})$ & $97,9 \mathrm{~dB}(\mathrm{~A})$ & $97,9 \mathrm{~dB}(\mathrm{~A})$ & $60 \mathrm{~dB}(\mathrm{~A})$ & $121,1 \mathrm{~dB}(\mathrm{~A})$ & $97,9 \mathrm{~dB}(\mathrm{~A})$ & $97,9 \mathrm{~dB}(\mathrm{~A})$ & $135,9 \mathrm{~dB}(\mathrm{~A})$ & $\begin{array}{l}\overline{8} 5 \overline{\mathrm{dB}}(\mathrm{A}) \overline{\mathrm{a}} \\
99,5 \mathrm{~dB}(\mathrm{~A})\end{array}$ & MEDIO \\
\hline & \multirow{2}{*}{ Cepillado/Canteado } & $\begin{array}{l}\text { Trabajador \#5 } \\
\text { Trabajador \#6 }\end{array}$ & Intermitente & 8 horas & $\begin{array}{l}85 \mathrm{~dB}(\mathrm{~A}) \\
----1\end{array}$ & $\begin{array}{r}96,3 \mathrm{~dB}(\mathrm{~A}) \\
-----\end{array}$ & $\begin{array}{l}95,7 \mathrm{~dB}(\mathrm{~A}) \\
-----\end{array}$ & $\begin{array}{c}63,1 \mathrm{~dB}(\mathrm{~A}) \\
-----\end{array}$ & $\begin{array}{l}117,3 \mathrm{~dB}(\mathrm{~A}) \\
--1---.\end{array}$ & $\begin{array}{r}95,7 \mathrm{~dB}(\mathrm{~A}) \\
-----\end{array}$ & $\begin{array}{l}96,3 \mathrm{~dB}(\mathrm{~A}) \\
----\end{array}$ & $\begin{array}{l}136,5 \mathrm{~dB}(\mathrm{~A}) \\
----\end{array}$ & $\begin{array}{r}85 \mathrm{~dB}(\mathrm{~A}) \mathrm{a} \\
99,5 \mathrm{~dB}(\mathrm{~A})\end{array}$ & MEDIO \\
\hline & & $\begin{array}{l}\text { Trabajador \#7 } \\
\text { Trabajador \#8 } \\
\end{array}$ & Intermitente & 8 horas & $85 \mathrm{~dB}(\mathrm{~A})$ & $97,1 \mathrm{~dB}(\mathrm{~A})$ & $97,1 \mathrm{~dB}(\mathrm{~A})$ & $63,1 \mathrm{~dB}(\mathrm{~A})$ & $119,5 \mathrm{~dB}(\mathrm{~A})$ & $96,4 \mathrm{~dB}(\mathrm{~A})$ & $97,1 \mathrm{~dB}(\mathrm{~A})$ & $138,5 \mathrm{~dB}(\mathrm{~A})$ & $\begin{array}{l}85 \mathrm{~dB}(\mathrm{~A}) \text { a } \\
99,5 \mathrm{~dB}(\mathrm{~A})\end{array}$ & MEDIO \\
\hline \multirow[t]{2}{*}{ Empresa \#3 } & Cepillado/Canteado & $\begin{array}{l}\text { Trabajador \#1 } \\
\text { Trabajador \#2 }\end{array}$ & Intermitente & 8 horas & $85 \mathrm{~dB}(\mathrm{~A})$ & $96,2 \mathrm{~dB}(\mathrm{~A})$ & $99,3 \mathrm{~dB}(\mathrm{~A})$ & $60 \mathrm{~dB}(\mathrm{~A})$ & $121,1 \mathrm{~dB}(\mathrm{~A})$ & $99,3 \mathrm{~dB}(\mathrm{~A})$ & $96,2 \mathrm{~dB}(\mathrm{~A})$ & $135,9 \mathrm{~dB}(\mathrm{~A})$ & $\begin{array}{l}85 \mathrm{~dB}(\mathrm{~A}) \mathrm{a} \\
99,5 \mathrm{~dB}(\mathrm{~A})\end{array}$ & MEDIO \\
\hline & Sierra Sinfin & $\begin{array}{l}\text { Trabajador \#3 } \\
\text { Trabajador \#4 } \\
\end{array}$ & Intermitente & 8 horas & $85 \mathrm{~dB}(\mathrm{~A})$ & $95,8 \mathrm{~dB}(\mathrm{~A})$ & $95,8 \mathrm{~dB}(\mathrm{~A})$ & $63,1 \mathrm{~dB}(\mathrm{~A})$ & $119,5 \mathrm{~dB}(\mathrm{~A})$ & $95,8 \mathrm{~dB}(\mathrm{~A})$ & $95,8 \mathrm{~dB}(\mathrm{~A})$ & $140,6 \mathrm{~dB}(\mathrm{~A})$ & $\begin{array}{l}85 \mathrm{~dB}(\mathrm{~A}) \mathrm{a} \\
99,5 \mathrm{~dB}(\mathrm{~A})\end{array}$ & MEDIO \\
\hline \multirow[b]{2}{*}{ Empresa \#4 } & Cepillado/Canteado & $\begin{array}{l}\text { Trabajador \#1 } \\
\text { Trabajador \#2 }\end{array}$ & Intermitente & 8 horas & $85 \mathrm{~dB}(\mathrm{~A})$ & $\begin{array}{r}96,8 \mathrm{~dB}(\mathrm{~A}) \\
-\end{array}$ & $96,99 \mathrm{~dB}(\mathrm{~A})$ & $\begin{array}{c}63,1 \mathrm{~dB}(\mathrm{~A}) \\
--\ldots--\end{array}$ & $118,1 \mathrm{~dB}(\mathrm{~A})$ & $\begin{array}{r}96,9 \mathrm{~dB}(\mathrm{~A}) \\
-\end{array}$ & $96,8 \mathrm{~dB}(\mathrm{~A})$ & $\begin{array}{l}136,6 \mathrm{~dB}(\mathrm{~A}) \\
--\ldots-\end{array}$ & $\begin{array}{l}85 \mathrm{~dB}(\mathrm{~A}) \mathrm{a} \\
99,5 \mathrm{~dB}(\mathrm{~A})\end{array}$ & $\begin{array}{r}\text { MEDIO } \\
-----\end{array}$ \\
\hline & Sierra Sinfin & $\begin{array}{l}\text { Trabajadoro \#3 } \\
\text { Trabajador \#4 }\end{array}$ & Intermitente & 8 horas & $85 \mathrm{~dB}(\mathrm{~A})$ & $96 \mathrm{~dB}(\mathrm{~A})$ & $96 \mathrm{~dB}(\mathrm{~A})$ & $60 \mathrm{~dB}(\mathrm{~A})$ & $112 \mathrm{~dB}(\mathrm{~A})$ & $96 \mathrm{~dB}(\mathrm{~A})$ & $96 \mathrm{~dB}(\mathrm{~A})$ & $138,9 \mathrm{~dB}(\mathrm{~A})$ & $\begin{array}{l}85 \mathrm{~dB}(\mathrm{~A}) \mathrm{a} \\
99,5 \mathrm{~dB}(\mathrm{~A}) \\
\end{array}$ & MEDIO \\
\hline $\begin{array}{c}\text { Nombres de las } \\
\text { Empresas }\end{array}$ & Lugar de Medición & $\begin{array}{l}\text { Nombres } \\
\text { Trabajadores }\end{array}$ & \begin{tabular}{|c|} 
Dosis \\
Encontrada \\
en \\
Porcentaje \\
\end{tabular} & $\begin{array}{c}\text { Dosis } \\
\text { Proyectada } \\
\text { Pdose }(8: 00)\end{array}$ & $\begin{array}{c}\text { Dosis } \\
\text { Permitida en } \\
\text { Porcentaje }\end{array}$ & DOSIS de RUIDO & $\begin{array}{l}\text { NIVEL DE } \\
\text { RIESGO }\end{array}$ & & & & & & & \\
\hline \multirow{2}{*}{ Empresa \#1 } & $\begin{array}{l}\text { Cepillado/Canteado } \\
-----~\end{array}$ & $\begin{array}{l}\text { Trabajador \#1 } \\
\text { Trabajador \#2 }\end{array}$ & $\begin{array}{l}4903,70 \% \\
----\end{array}$ & $\begin{array}{r}4783,40 \% \\
-----\end{array}$ & $\begin{array}{c}100 \% \\
----\end{array}$ & $\begin{array}{c}>3200 \% \\
-----.\end{array}$ & $\begin{array}{l}\text { ALTO } \\
----\ldots\end{array}$ & & & & & & & \\
\hline & Sierra Sinfin & $\begin{array}{l}\text { Trabajador \#3 } \\
\text { Trabajador \#4 }\end{array}$ & $1299,20 \%$ & $1265,50 \%$ & $100 \%$ & $100 \%$ a $3199 \%$ & MEDIO & & & & & & & \\
\hline \multirow{4}{*}{ Empresa \#2 } & 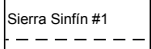 & $\begin{array}{l}\text { Trabajador \#1 } \\
\text { Trabajador \#2 }\end{array}$ & $\begin{array}{l}476,80 \% \\
---\end{array}$ & $\begin{array}{l}600,50 \% \\
---\end{array}$ & $\begin{array}{c}100 \% \\
---\end{array}$ & \begin{tabular}{|c|}
$100 \%$ a $3199 \%$ \\
-----
\end{tabular} & $\begin{array}{l}\text { MEDIO } \\
--\ldots\end{array}$ & & & & & & & \\
\hline & Sierra Sinfin \#2 & \begin{tabular}{|l} 
Trabajador \#3 \\
Trabajador \#4 \\
\end{tabular} & $1977,70 \%$ & $1956,40 \%$ & $100 \%$ & $100 \%$ a $3199 \%$ & MEDIO & & & & & & & \\
\hline & Cepillado/Canteado & $\begin{array}{l}\text { Trabajador \#5 } \\
\text { Trabajador \#6 } \\
\text { Trabajador } \# \text { \#7 }\end{array}$ & $\mid \begin{array}{c}1200,40 \% \\
-----\end{array}$ & $\begin{array}{l}1356,70 \% \\
----\end{array}$ & $\begin{array}{c}100 \% \\
----\end{array}$ & \begin{tabular}{|c|}
$100 \%$ a $3199 \%$ \\
------
\end{tabular} & $\begin{array}{l}\text { MEDIO } \\
-----\end{array}$ & & & & & & & \\
\hline & & $\begin{array}{l}\text { Iriabajador \# } \\
\text { Trabajador \#8 } \\
\end{array}$ & $1384,80 \%$ & $1647,10 \%$ & $100 \%$ & $100 \%$ a $3199 \%$ & MEDIO & & & & & & & \\
\hline \multirow{2}{*}{ Empresa \#3 } & Cepillado/Canteado & $\begin{array}{l}\text { Trabajador \#1 } \\
\text { Trabajador \#2 }\end{array}$ & 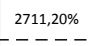 & $\begin{array}{l}1343,00 \% \\
---\end{array}$ & $\begin{array}{c}100 \% \\
----\end{array}$ & 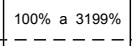 & MEDIO & & & & & & & \\
\hline & Sierra Sinfin & $\begin{array}{l}\text { Trabajajor \#3 } \\
\text { Trabajador \#4 }\end{array}$ & $1210,20 \%$ & $1203,40 \%$ & $100 \%$ & $100 \%$ a $3199 \%$ & MEDIO & & & & & & & \\
\hline \multirow{2}{*}{ Empresa \#4 } & Cepillado/Canteado & $\begin{array}{l}\text { Trabajador \#1 } \\
\text { Trabajador \#2 }\end{array}$ & $\begin{array}{l}1582,00 \% \\
----\end{array}$ & $\begin{array}{l}1540,60 \% \\
-\ldots--\end{array}$ & $100 \%$ & $\begin{array}{c}100 \% \text { a } 3199 \% \\
---\end{array}$ & $\begin{array}{l}\text { MEDIO } \\
-\ldots-\ldots\end{array}$ & & & & & & & \\
\hline & Sierra Sinfin & $\begin{array}{l}\text { Trabajajador \#3 } \\
\text { Trabajador \#4 }\end{array}$ & $1277,50 \%$ & $1262,30 \%$ & $100 \%$ & $100 \%$ a $3199 \%$ & MEDIO & & & & & & & \\
\hline
\end{tabular}

Fuente: Investigadores

En las operaciones de cepillado/canteado de la empresa 1, presenta el nivel continuo equivalente de ruido más crítico, (ver Tabla 1). Por tal razón, se realizó el Análisis de Frecuencias, con el fin de conocer cómo se distribuye la energía acústica entre las diferentes frecuencias de bandas en octavas (su distribución espectral de ruido) presentando niveles de energía sonora más altos en las frecuencias altas $(2000 \mathrm{~Hz}$ a $4000 \mathrm{~Hz})$, capaces de producir una alta probabilidad de daños en la salud auditiva de los trabajadores expuestos, Figura 1. Este análisis nos permitió determinar el protector auditivo más eficaz a nivel de prevención de la hipoacusia neurosensorial inducido por ruido. 


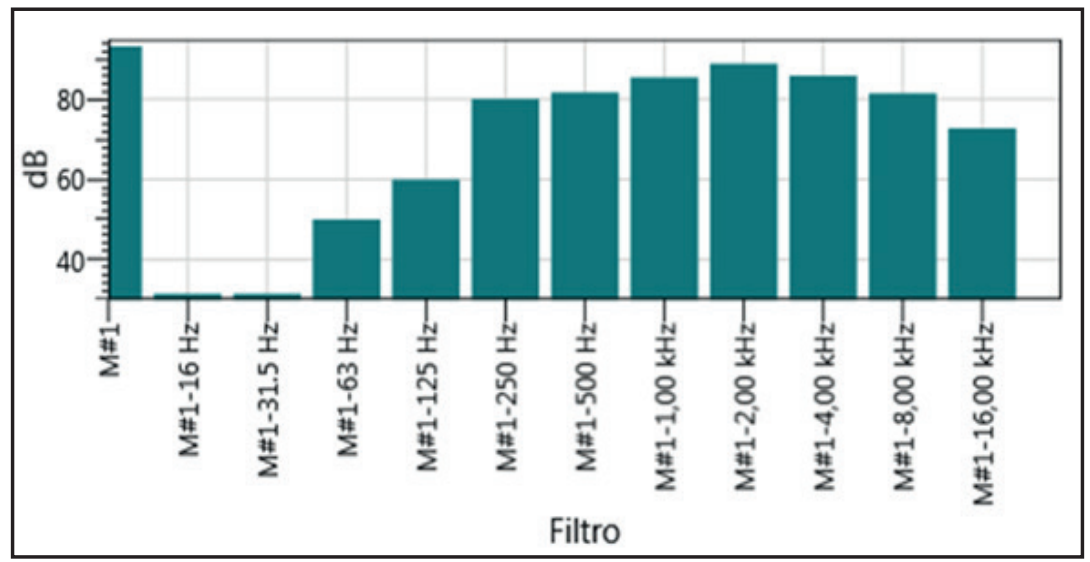

Figura 1. Análisis de Frecuencia

Fuente: Investigadores

\section{Diagnóstico de bipoacusia neurosensorial inducida por mido.}

En los trabajadores expuestos al ruido en las em- de Cartagena, presentaron el 20\% de hipoacusia presas seleccionadas del sector madera de la ciudad en los diferentes puestos de trabajo así:

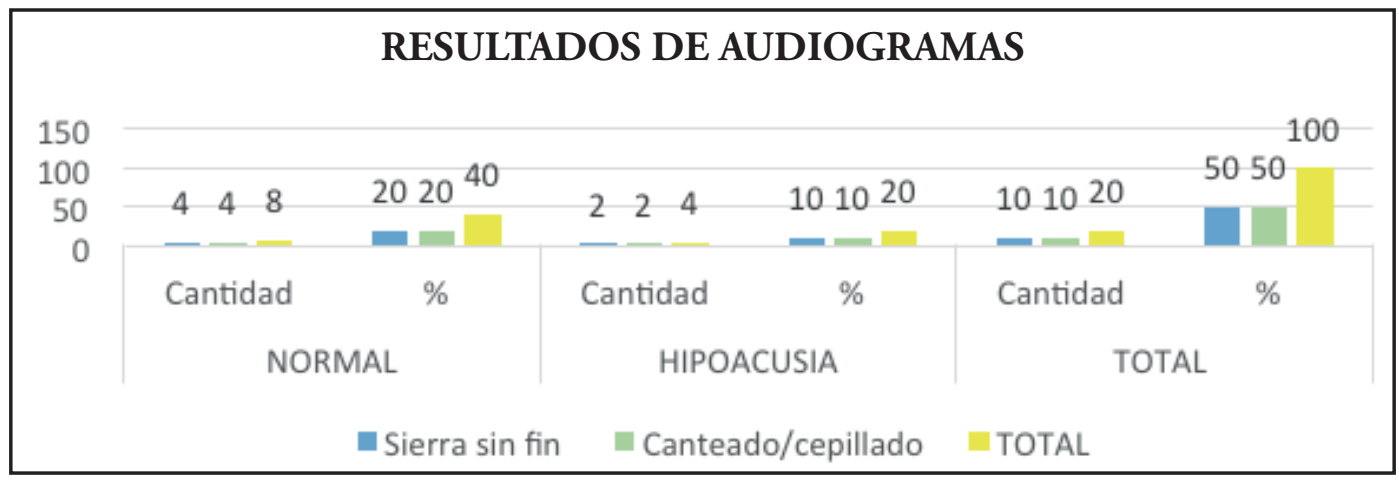

Figura 2. Hipoacusia vs puesto de trabajo

Fuente: Autores

Tabla 2. Hipoacusia vs puesto de trabajo

\begin{tabular}{|c|c|c|c|c|}
\hline $\begin{array}{c}\text { HIPOACUSIA } \\
(\mathrm{CIE}-10 ; \mathrm{H} 91,9) \text { NIOSH }\end{array}$ & $\begin{array}{l}\text { SIERRA } \\
\text { SIN FIN }\end{array}$ & $\begin{array}{l}\text { CANTEADORAS } \\
\text { CEPILLADORA }\end{array}$ & TOTAL & HIPOACUSIA \\
\hline Normal & 8 & 8 & 16 & 80 \\
\hline Leve & 0 & 1 & 1 & 5 \\
\hline Moderada & 1 & 0 & 1 & 5 \\
\hline Moderada a severa & 0 & 0 & 0 & 0 \\
\hline Severa & 0 & 0 & 0 & 0 \\
\hline Profunda & 1 & 1 & 2 & 10 \\
\hline Total & 10 & 10 & 20 & 100 \\
\hline
\end{tabular}

Fuente: Autores 
El 5\% de los trabajadores presentaron hipoacusia es leve, un 5\% con hipoacusia moderado y un
$10 \%$ con hipoacusia profunda de acuerdo a los criterios de la NIOSH. Según Tabla 3.

Tabla 3. Edad vs hipoacusia

\begin{tabular}{|c|c|c|c|c|c|c|}
\hline \multirow{3}{*}{ GRUPOS DE EDADES } & \multicolumn{6}{|c|}{ RESULTADO DE AUDIOGRAMAS } \\
\hline & \multicolumn{2}{|c|}{ NORMAL } & \multicolumn{2}{|c|}{ HIPOACUSIA } & \multicolumn{2}{|c|}{ TOTAL } \\
\hline & Cantidad & $\%$ & Cantidad & $\%$ & Cantidad & $\%$ \\
\hline Menor de 25 años & 1 & 5 & 0 & 0 & 1 & 5 \\
\hline 26 a 30 años & 1 & 5 & 0 & 0 & 1 & 5 \\
\hline 31 a 35 años & 2 & 10 & 0 & 0 & 2 & 10 \\
\hline 35 a 40 años & 3 & 15 & 0 & 0 & 3 & 15 \\
\hline 41 a 45 años & 3 & 15 & 1 & 5 & 4 & 20 \\
\hline Más de 46 años & 6 & 30 & 3 & 15 & 9 & 45 \\
\hline TOTAL & 16 & 80 & 4 & 20 & 20 & 100 \\
\hline
\end{tabular}

Fuente: Investigador

Un 5\% de los trabajadores que presentaron hipoacusia, tienen edades entre los 41 y 45 ańos de edad y un 15\% de los trabajadores con hipoacusia tienen más de 46 años, siendo este último grupo atáreo es el de mayor afectación. Todos los trabajadores expuestos son del género masculino. Se demuestra que los casos registrados en este estudio tienen como característica las edades más avanzadas y periodos largos de exposición.

\section{Discusión}

En la literatura internacional sobre el ruido éste se ha identificado como un contaminante presente en las distintas empresas, en especial aquellas donde se realiza trasformación y/o destrucción de la materia prima para la generación de productos terminados o de otras cadenas productivas. Por esto, se ha generado gran interés en relación a cómo el ruido en sus distintas intensidades puede contribuir a la aparición de enfermedades ocupacionales, quebrantando la salud de los trabajadores que se exponen diariamente a este riesgo en sus distintos centros de trabajo, por lo cual se hace indispensable analizar el fenómeno de las afectaciones auditivas condicionadas por la exposición al ruido en tiempos prolongados.

En un estudio sobre ruido en empresas del sector madera del parque industrial de la ciudad de Cuenca en el área de maquinado, Edgar Pozo Andrade analizó tres empresas (que identificó como alfa, beta y gamma) (3). En la empresa alfa los niveles de ruido oscilaron entre $73 \mathrm{db}(\mathrm{A})$ a 84,5 $\mathrm{dB}(\mathrm{A})$, en ningún puesto de trabajo sobrepasaron los valores límites permisibles de $85 \mathrm{~dB}(\mathrm{~A})$. En la empresa Beta, en el área de maquinado, el nivel equivalente fue de $82,5 \mathrm{~dB}(\mathrm{~A})$ a $91,2 \mathrm{~dB}(\mathrm{~A})$, en la sierra múltiple, el torneado y la perfiladora sobrepasan el límite máximo permisible de $85 \mathrm{~dB}(\mathrm{~A})$ según los estándares y normas nacionales. En la empresa gamma, en el área de maquinado el nivel equivalente fue de $91,5 \mathrm{~dB}(\mathrm{~A})$ a $96,4 \mathrm{~dB}(\mathrm{~A})$, apreciándose que, en la cepilladora, la sierra circular, el torneado, el lijado y el ensamblado sobrepasan el límite máximo permisible según los estándares y normas nacionales estipuladas. En el anterior estudio los trabajadores utilizan protección auditiva tipo tapón. En contraste al anterior estudio, la 
prevalencia de hipoacusia neurosensorial inducida por ruido en cuatro empresas del sector madera de la ciudad de Cartagena, los niveles equivalentes diarios que se originan en las operaciones de maquinado sobrepasan los valores límites permisibles de $85 \mathrm{~dB}(\mathrm{~A})$ de acuerdo los criterios de ACGIH de USA, existiendo además aspectos no mencionados en el estudio, la prevalencia de hipoacusia y el estudio sociodemográfico de la población expuesta al ruido; por lo tanto, no se podría correlacionar con resultados del presente estudio.

Hernández y González (7), en su estudio sobre determinación del grado de afección por ruido en los trabajadores en la empresa Carpintería de Aluminio “Tomás Álvarez Breto", situada en la Ciudad de La Habana, encontraron que los niveles de ruido superaba los niveles de seguridad de $85 \mathrm{db}(\mathrm{A})$ en 9 de los 13 departamentos con que cuenta el centro y ha afectado la salud de los trabajadores, ya que existían 77 casos $(78,5 \%)$ de hipoacusia atribuible al ruido y un gran número de trabajadores $(30,6 \%)$ expuestos a elevados niveles de ruido innecesariamente por la naturaleza de su labor. El ruido ha afectado la salud de los trabajadores de este centro, ya que se encontraron 77 casos de hipoacusia atribuible al ruido, lo que representó el $78,5 \%$ del total estudiado; mientras que en el estudio en las empresas del sector de madera en la ciudad de Cartagena, los niveles equivalentes diarios que se originan en las operaciones de maquinado sobrepasan los valores límites permisibles de $85 \mathrm{~dB}(\mathrm{~A})$ de acuerdo los criterios de ACGIH de USA; coincidiendo con los resultados obtenidos por Hernández y González con relación a la exposición al ruido.

En lo referente a la prevalencia de hipoacusia en los trabajadores expuestos al ruido estudiados fue del $78,5 \%$, siendo muy alto en relación a la prevalencia de los trabajadores expuestos al ruido en las empresas del sector madera en la ciudad de Cartagena, alrededor del $20 \%$ con más de 41 años de edad. Es importante resaltar que en el estudio de los cubanos, Hernández y González no establecieron la relación de la prevalencia con respecto al tiempo de servicio y la edad de los trabajadores; aspecto diferenciador en el estudio realizado en Cartagena.
Estudios como los adelantados por Moreno, Martínez y Rivero (8), establecen que la contaminación sonora y el daño auditivo es inherente a las diversas actividades que desarrolle la empresa y la omisión de las recomendaciones y protocolos higiénicos tendientes a la protección de la sordera ocupacional puede perjudicar la audición debido a la exposición a altos índices de emisiones acústicas contaminantes. Asimismo, en el actual estudio se encontraron exposiciones nocivas a niveles de ruido por encima de $85 \mathrm{~dB}(\mathrm{~A})$ con alta posibilidad de dańo auditivo. Tanto en (8) como en el presente estudio se considera la variable "años de exposición” como generador de pérdida auditiva, determinando así la relación directa del daño auditivo versus tiempo de servicio.

Hay otro estudio sobre una prevalencia de la pérdida auditiva en una fábrica en Nepal, en un nivel de $28 \%$ entre los trabajadores que desarrollaban actividades de sierra sin fin, con una exposición de ruido promedio $90.2 \mathrm{~dB}$ (10). En cuanto a la prevalencia de hipoacusia de los trabajadores en las empresas del sector madera en Cartagena se halla un nivel inferior $20 \%$ siendo menor a la prevalencia de los trabajadores expuestos a ruido en la empresa referenciada inicialmente, diferenciando el daño auditivo con los niveles de ruido por encima de los permisibles, el tiempo de servicio y la edad, como en el presente estudio de las empresas del sector madera de la ciudad de Cartagena.

Zamorano et al. (11) determinaron la prevalencia de la disminución auditiva entre 164 trabajadores expuestos a ruido en una empresa metalmecánica de los cuales el 53\% de los trabajadores presentaba una audición normal el 53\% de los trabajadores con una audición normal, mientras que la disminución auditiva tuvo lugar en un $47 \%$ de los trabajadores. Las zonas de trabajo presentaron niveles de ruido en un rango de 83 a 102 decibeles. El contar con más edad y mayor antigüedad no influyó en la disminución de la capacidad auditiva. Sugirieron ampliar el estudio con más antecedentes personales y datos extra-laborales que permitiese conocer mejor a la población. 
En el presente estudio, la prevalencia de hipoacusia en los trabajadores expuestos a ruido es menor a la prevalencia de los trabajadores expuestos a ruido en la empresa metalmecánica; coincidiendo en los niveles de ruido que se encuentran por encima de los valores de referencia.

Concordante con el estudio de Alonso Díaz (13), donde las lesiones auditivas por ruido ocupacional dañan la posibilidad de mantener conversaciones en detrimento de la calidad de vida de las personas causado por los prolongados tiempos de exposición y la edad. Con semejanzas en el desarrollo de los eventos de salud y detrimento entre los trabajadores positivos para hipoacusia en empresas del sector madera en Cartagena al superar los niveles permisibles, entra a ser relevante indicar que en ambos estudios se tuvo en cuenta las variables de edad y tiempo de servicio.

Como conclusión, las empresas seleccionadas del sector madera de la ciudad de Cartagena, los trabajadores se exponen a ruido de tipo intermitente y variado durante la jornada laboral de 8 horas diarias y 48 horas semanales. Las empresas no tienen ningún sistema de control de ingeniería del ruido en las distintas fuentes, los trabajadores no utilizan ningún protector auditivo al exponerse al ruido en las distintas tareas del proceso de aserrío.

Los niveles diarios equivalentes y los niveles de pico del ruido que se generan en las distintas máquinas superan los $85 \mathrm{~dB}(\mathrm{~A})$, niveles de referencia de acuerdo con los criterios de la ACGIH de USA.

La prevalencia de hipoacusia neurosensorial inducida por ruido es del $20 \%$ en los trabajadores expuestos. $15 \%$ con hipoacusia leve y un 5\% con hipoacusia moderada de acuerdo a la clasificación de la NIOSH.

La prevalencia de perdida auditiva esta relacionada con mayor edad en los trabajadores en las empresas en estudio; mientras Zambrano et al (11) obtuvo en su estudio que la variable edad no determinó afectación auditiva en sus trabajadores.

\section{Recomendaciones}

Las empresas seleccionadas del sector Madera en la ciudad de Cartagena deben implementar un programa de conservación auditiva que tiene como objetivo general prevenir y controlar la aparición de la pérdida auditiva inducida por la exposición a ruido ocupacional. El programa de conservación auditiva debe estar dirigido a los trabajadores permanentes y temporales en las empresas.

Se debe establecer un programa de mantenimiento preventivo y correctivo en forma periódica en las distintas fuentes del ruido.

Establecer un programa de elementos de protección auditiva que incluya la selección de protectores teniendo en cuenta el certificado de calidad y el análisis de suficiencia de atenuación, inspecciones, mantenimiento y reposición, capacitación y entrenamiento para uso del protector auditivo.

Implementar un programa de vigilancia médica que incluya a todos los trabajadores expuestos a niveles iguales o superiores a $80 \mathrm{dBA}$ TWA. Los programas deben contener evaluaciones médicas, aplicación de cuestionarios tamiz, y pruebas audiológicas.

Las evaluaciones médicas deben realizarse cada año para los trabajadores expuestos a niveles de ruido de 82 a 99 dBA TWA. (Ministerio de Protección Social. Guía de atención integral basadas en la evidencias para hipoacusia neurosensorial por ruido en el lugar de trabajo. 2006).

Capacitar a los trabajadores sobre la importancia del uso de la protección auditiva y realizar una supervisión de estos y la utilización de "zonas de protección auditiva obligatoria" adecuadamente definidas, delimitadas y señalizadas.

Evitar conductas de riesgo auditivo en ambientes extra-laborales. 


\section{Referencias}

1. Marx H, Rosario E. Manual de las Enfermedades del Oído. 6ta. edición. Madrid: Calpe S.A; 2007. p. 12-8.

2. Le Prell C G, Dolan D F, Schacht J, Miller J M, Lomax M I, Altschuler R A. Pathways for protection from noise induced hearing loss. Noise Health 2003; 5:1-17.

3. Pozo Andrade, E. Estudio de ruido generado en la industria maderera en la ciudad de Cuenca y sus efectos a la salud [Maestría]. Universidad de Cuenca 2010.p. 14-15.

4. Otárola Merino F, Otárola Zapata2 F, Finkelstein Kulka A. Ruido Laboral y su Impacto en Salud. Ciencia y Trabajo. 2006; 8 (20):47-51.

5. Gómez Martínez M, Jaramillo García J, Luna Ceballos Y, Martínez Valencia A, Velásquez Zapata M, Vásquez E. Ruido industrial: efectos en la salud de los trabajadores expuestos. Revista CES. 2012; 3(2):174-183.

6. Gómez Aconcha S, Pirazan Martínez J, Alexa S. Proyecto de promoción de salud auditiva y prevención de pérdidas auditivas para empleados de Mecars impresores de la localidad de Kennedy en la ciudad de Bogotá [Pregrado]. Corporación Universitaria Iberoamericana; 2012.

7. Hernández Díaz A, González Méndez B. Alteraciones auditivas en trabajadores expuestos al ruido industrial. Medicina $y$ seguridad del trabajo. 2007;53(208):2-3.

8. Moreno Rajadel René Esteban, Martínez Díaz Anay, Rivero Pérez Diamelys. Pesquisa auditiva en trabajadores expuestos al ruido industrial. Rev. Cubana Med Gen Integr [Internet]. 2006 Sep [citado 2015 Mar 20]; 22(3): Disponible en: http:// scielo.sld.cu/scielo.php?script=sci_arttext \&pid=S086421252006000300003\&lng=es

9. Santana-Herrera José, Alfano Tony, Escobal-Machado Adriana. Turnos de trabajo: ¿un factor de riesgo cardiovascular? Med. segur. trab. [Internet]. 2014 Mar [citado 2015 Abr 9]; 60(234): 179-197. Disponible en: http:// scielo.isciii.es/scielo.php?script=sci_arttext\&pid=S0465546X2014000100014\&lng=es. http://dx.doi.org/10.4321/S0 465-546X2014000100014.

10. Dhere AM, Pawar CB, Patil DA, Pawar JA. Noise induced hearing loss (NIHL) in saw mill and printing press workers in Akluj Town of Solapur district. J Environ Sci Eng. 2009 Jul; 51(3):187-190

11. Zamorano González B, Parra Sierra V, Vargas Martínez JI, Castillo Muraira Y, Vargas Ramos C. Disminución auditiva de trabajadores expuestos a ruido en una empresa metalmecánica. Ciencia \& Trabajo; 2012; 35: 233-236.

12. Medina Medina A, Velásquez Gómez G, Giraldo Vargas L, Henao Ayora L, Vásquez Trespalacios E. Sordera ocupacional: una revisión de su etiología y estrategias de prevención. CES Salud Pública. 2013; 4(2):116-124.
13. Alonso Díaz, J. Resultados de la aplicación del protocolo de ruido en trabajadores expuestos a un nivel de ruido continuo diario equivalente igual o superior a 85 decibelios (A). Med. segur. trab. [Internet]. 2014 Mar [citado 2015 Oct 11]; 60(234): 9-23. Disponible en: http://scielo.isciii.es/scielo.php?script=sci_arttext\&pid=S0465-546X2014000100003\&lng=es. http:// dx.doi. org/10.4321/S0 465-546X2014000100003.

14. Ugalde López A, Dolci Fajardo G. Hipoacusia por ruido: Un problema de salud y de conciencia pública. abril de 2000; Rev. Fac Med UNAM Vol.43 marzo-abril, 2000(No.2). Recuperado a partir de: http:// www.ejournal.unam.mx/rfm/no43-2/ RFM43202. Pdf

15. Colombia. Ministerio de la Protección Social. Guía de atención integral de salud ocupacional basada en la evidencia para hipoacusia neurosensorial inducida por ruido en el trabajo. Sitio web de www.minsalud.gov.co. www.minsalud.gov.co. [En línea] 16 de diciembre de 2006. [Citado el: 3 de marzo de 2016.] www.minsalud.gov.co/Documentos\%20y\%20Publicaciones/ GATISO-HIPOACUSIA\%20NEROSENSORIAL.pdf.

16. Flores Hernández, Corina; Huerta Franco, Raquel; Carrillo Soto, José Guadalupe; Zarate Vera, Teresa; Mc-Grath Bernal, Mary Jean; Morales Mata, Ismael. Incidencia de estrés en odontólogos de diferentes especialidades ocasionado por ruido en el consultorio dental. Nova Scientia. 2009;1(2):1-21.

17. Fernández-D’Pool J, Butrón J, Colina-Chourio J. Efecto del ruido sobre la presión arterial en trabajadores de una empresa petrolera venezolana. Investigación Clínica. 2010;51(3):301-314.

18. Stewart, M, Koltes, K y Lehman, M. Noise Exposure Levels for Workers in the Michigan Wood Industry. Michigan: Consultation Education and Training (CET), 2005.

19. Castro Maria Euridéa de, Rolim Maysa Oliveira, Mauricio Tibelle Freitas. Prevenção da hipertensão e sua relação com o estilo de vida de trabalhadores. Acta paul. enferm. [Internet]. 2005 June [cited 2015 Apr 12] ; 18( 2 ): 184-189. Available from: http://www.scielo.br/scielo.php?script=sci_arttext\&pid =S0103-21002005000200011\&lng=en. http://dx.doi.org/10. 1590/S0103-21002005000200011.

20. Millan, J. and L. Yunda (2014). "An Open-Access Web-based medical image atlas for collaborative Medical image sharing, processing, Web Semantic searching and analysis with uses in medical training, research and second opinion of cases." Nova 12(22): 143-150. 